\title{
Z-copy Voltage Controlled Current Follower Differential Input Transconductance Amplifier in Controllable Biquadratic Band-Pass Filter
}

\author{
Roman Sotner ${ }^{1}$, Jan Jerabek ${ }^{2}$, Tomas Dostal ${ }^{3}$, Kamil Vrba ${ }^{2}$ \\ ${ }^{I}$ Department of Radio Electronics, Faculty of Electrical Engineering and Communication, \\ Brno University of Technology, \\ Technicka 12, Brno, 616 00, Czech Republic \\ ${ }^{2}$ Department of Telecommunications, Faculty of Electrical Engineering and Communication, \\ Brno University of Technology, \\ Technicka 12, Brno, 616 00, Czech Republic \\ ${ }^{3}$ Department of Technical Studies, College of Polytechnics Jihlava, \\ Tolsteho 16, Jihlava 586 01, Czech Republic \\ sotner@feec.vutbr.cz
}

\begin{abstract}
This paper presents the results of study on utilization of Z-copy Voltage Controlled Current Follower Differential Input Transconductance Amplifier (ZCVCCFDITA) active element in a single purpose filtering structure. This active element has three voltage-controlled parameters (intrinsic resistance of current input, voltage gain and transconductance of output section) and is very useful for design of oscillators, signal generators and frequency filters with just one active element. In this paper, the important parameters of possible behavioural model of ZC-VCCFDITA are shown and a particular solution of the controllable bandpass filter is presented. Theoretical, calculated, simulated and moreover also measured results are mutually compared.
\end{abstract}

Index Terms - Analog behavioural modelling; band-pass filter; current conveyor; current follower; controllable input resistance; current inverter; electronic adjusting (control); transconductance; Z-copy voltage controlled current follower differential input transconductance amplifier.

\section{INTRODUCTION}

In the recent decades, extreme attention has been given to electronically controllable multifunctional or universal active filter proposal (for example [1]-[10]). Many of the reported structures allow electronic control of the key features of the filter (adjusting of pass-band gain, pole frequency $\omega_{0}$ and quality factor $Q$ ) [1]-[3], [6]-[10]. However, some structures require the increasing number of active devices in order to obtain multifunctionality and adjustability simultaneously [1]-[3]. There are some really very simple solutions of the multifunctional filters (for example [11]-[13]) but electronic control of their

Manuscript received 21 December, 2015; accepted 28 March, 2016.

Research described in this paper was financed by Czech Ministry of Education in frame of National Sustainability Program under grant LO1401. For research, infrastructure of the SIX Center was used. Research described in the paper was supported by Czech Science Foundation projects under No. 14-24186P. parameters is problematic or not possible. Some communication subsystems in base- or inter-frequency- band need specific transfer response only. Band-pass (BP) filtering response is one of the most significant transfer functions in inter-frequency bands (hundreds of $\mathrm{kHz}$, units and tens of $\mathrm{MHz}$ ) of radiofrequency subsystems, especially if their parameters can be adjusted electronically. It is important for measuring instruments (selective voltmeters) especially, inter-frequency filters of radio-communication receivers, transmitters, etc.

In recent years, differential active devices and filtering structures ([14]-[18] for example) have achieved substantial attention especially due to their better suppression of noise and common mode distortion [14]. Development of electronically controllable BP solutions focused on differential filtering structures. Unfortunately, increasing complexity of fully-differential circuitry and also necessity of asymmetric (single-ended) signal processing (singleended signal processing operations prevail in almost all subsystem) leads to search of some beneficial, very simple and single-ended structures in many cases. Modern active devices [19], consisting of several active subparts, allow easier kind of circuit synthesis (simple structure utilizing only one or two active devices). In addition, it brings further simplification of overall circuitry if only one or only few transfer responses are sufficient for target application in comparison to completely universal systems (for example [1]-[10]). Electronically controllable $\left(Q\right.$ adjusting and $\omega_{0}$ tuning) features of the single BP response were studied except its availability in multifunctional/universal structures. Several band-pass filtering structures available in recent literature are summarized in Table I in order to compare the achievable features.

In fact, there were not very similar BP filtering circuits proposed in the past (for example [20] but in part of a multifunctional solution), especially not many structures 
allowing simple single-parameter control of $Q$ (similar to selected multifunctional biquadratic structures [1]-[3]). Practical implementations of BP response available from multiple-loop state-variable [1], [4] structure requires electronic control of two parameters simultaneously for bandwidth change of the BP response in many cases [21]. It is not the case of the presented solution (only one-parameter adjusting used).

TABLE I. BRIEF COMPARISON OF RECENTLY REPORTED

\begin{tabular}{|c|c|c|c|c|c|c|c|}
\hline \multirow[b]{2}{*}{ 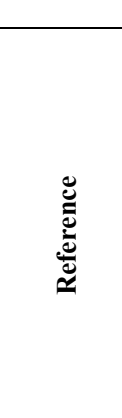 } & \multicolumn{7}{|c|}{ ADJUSTABLE BP SOLUTIONS. } \\
\hline & 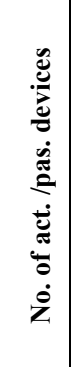 & 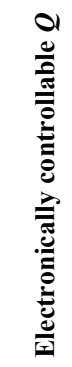 & 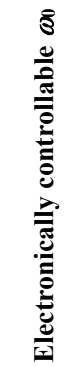 & 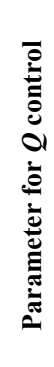 & 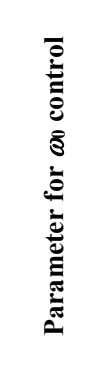 & 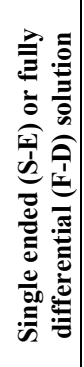 & 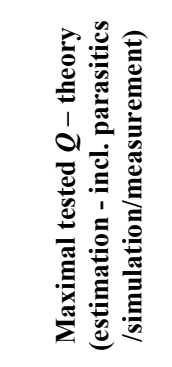 \\
\hline$[17]$ & $2 / 10$ & No & No & - & - & F-D & N/A \\
\hline [22] & $3 / 4$ & Yes & No & $B$ & - & S-E & $6(-/ 4 /-)$ \\
\hline [22] & $5 / 6$ & Yes & No & $B$ & - & F-D & $6(-/ 4.5 /-)$ \\
\hline [23] & $4 / 4$ & Yes & No & $B$ & - & S-E & $3(-/ 2.8 /-)$ \\
\hline$[23]$ & $7 / 4$ & Yes & No & $B$ & - & F-D & $4.1(-/ 3.7 /-)$ \\
\hline proposed & $1 / 2$ & Yes & Yes & $A$ & $g_{\mathrm{m}} / R_{\mathrm{f}}$ & S-E & $80(62 / 45 / 50)$ \\
\hline
\end{tabular}

Some of the modern active devices reported in [19], allowing electronic control of their parameters, are very useful for utilization in these applications. Presented Z-Copy Voltage Controlled Current Follower Differential Input Transconductance Amplifier (ZC-VCCFDITA) [24], [25] belongs to this group. Inter-terminal controllable relations predetermine this active device for various simple externally controllable applications and are very useful also for design of electronically controllable BP structure.

This paper is divided into six sections. The introductory section deals with recent development of BP filters and presents a comparison of the features available in literature and the target features of the solution discussed in this paper. The second section explains a principle of operation of used active device. Section III discusses ideal behaviour of the proposed BP filter. Non-ideal analysis is provided in Section IV. The results of simulations and measurements are evaluated in Section $\mathrm{V}$ and the most important features of the structure are summarized in Section VI.

\section{BRIEF DESCRIPTION OF ZC-VCCFDITA PRINCIPLE}

Despite full information given in [24], a brief description of active device is provided in order to make further discussions clearer and understandable. Z-Copy Voltage Controlled Current Follower Differential Input Transconductance Amplifier (ZC-VCCFDITA) presented in this paper was derived (minor modification) from the $\mathrm{ZC}$ VCCFDITA in [24] and its ideal behavior is also very similar to a principle of Generalized Current Follower Differential Input Transconductance Amplifier (GCFDITA) [26] and family of active devices discussed in [27]. The ZCVCCFDITA offers the most important benefits in its fully independent multiple-parameter electronical controllability, namely: resistance of current input terminal $\left(R_{\mathrm{f}}\right)$ [28], [29]; transconductance $\left(g_{\mathrm{m}}\right)$ [30] and stage of voltage amplifier (A) [31]. A principle explaining ideal behavior of the $\mathrm{ZC}$ VCCFDITA concept is illustrated in Fig. 1.

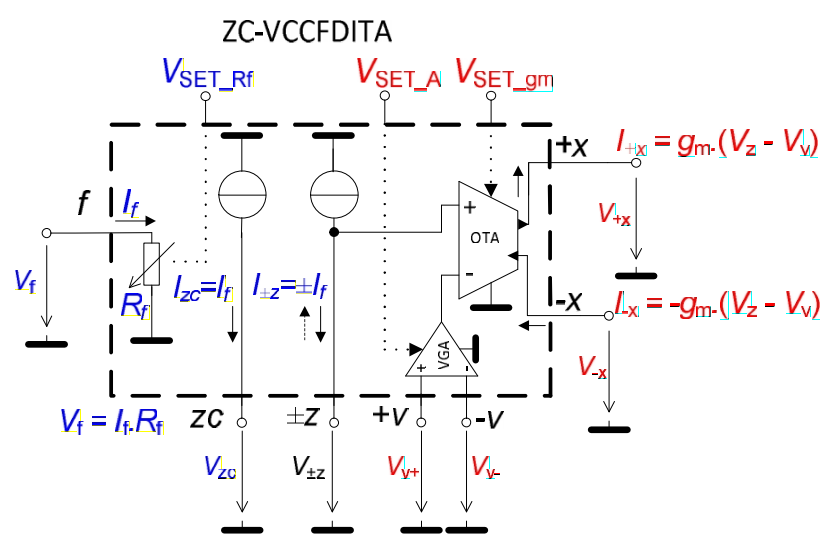

Fig. 1. Behavioural (ideal, linear) model of Z-copy voltage controlled current follower differential input transconductance amplifier.

Two internal sub-parts (current follower/inverter with controllable input resistance and enhanced transconductance stage) create the main core of the device. In practice, both these subparts are integrated together in future case of its onchip implementation. Current $I_{\mathrm{f}}$ that is flowing to the lowimpedance input terminal (f) with the feature of controllable resistance $\left(R_{\mathrm{f}}\right)$, is mirrored (directly or inverted) to terminals $z$ and $z c$ (auxiliary copy of $I_{\mathrm{f}}$ ). These operations are clear from following expressions:

$$
\begin{gathered}
I_{+z}=-I_{-z}=I_{z c}=I_{f}, \\
V_{f}=I_{f} R_{f} .
\end{gathered}
$$

Simultaneously, terminal $\pm z$ creates also positive input of the transconductance section that follows after current inverter/follower part. Note that both voltage inputs (positive and negative) of the transconductance section are available in comparison to standard CFTA definition [19]. Our modification has this terminal fixedly connected to the output of variable gain amplifier (VGA) with two voltage inputs marked as $+v$ and $-v$ that are also inputs of the whole ZC-VCCFDITA block. Output current $I_{ \pm \mathrm{x}}$ is created in several steps. Voltage difference of $+v$ and $-v$, multiplied by adjustable value of $A$ (voltage gain) is subtracted by voltage on $\pm z$. Transformation of voltage difference at the input of the transconductance section to current $I_{ \pm_{\mathrm{x}}}$ is provided by $g_{\mathrm{m}}$

$$
I_{+x}=-I_{-x}=g_{m}\left(V_{ \pm z}-A\left(V_{+v}-V_{-v}\right)\right)
$$

The behavioural model of ZC-VCCFDITA has been presented in [24] and [25] (together with relevant equations and its features obtained from the simulations). Just for quick reference, it consists of several commercially available active elements and it provides basic function of $\mathrm{ZC}$ VCCFDITA with certain limits of tuning derived from respective limitations of these real devices that form this behavioural model. Note that this model has bandwidth of inter-terminal transfers approximately up to $20 \mathrm{MHz}$. 


\section{IDEAL BEHAVIOUR OF THE DESIGNED FILTER}

Designed resistor-less filtering structure with controllable features with just one ZC-VCCFDITA, two grounded capacitors and one additional voltage buffer that is not necessary if the following section has high input impedance, is presented in Fig. 2.

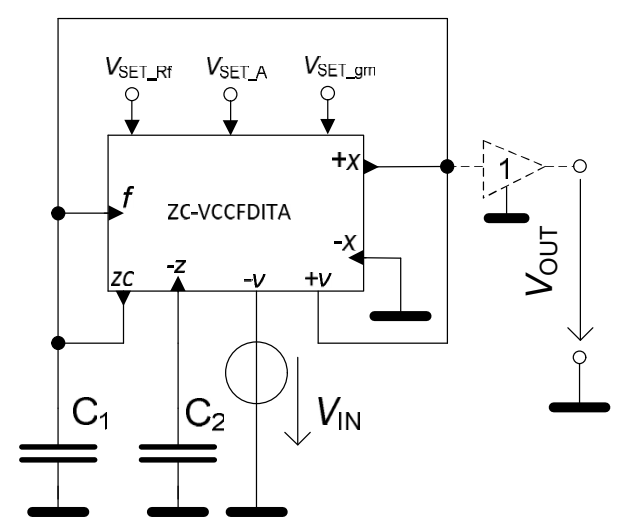

Fig. 2. Designed controllable resistor-less band-pass filter operating in the voltage mode with one ZC-VCCFDITA.

Ideal voltage-mode band-pass (BP) transfer function of the circuit in Fig. 1 is following

$$
K_{B P}(s)=\frac{V_{O U T}}{V_{I N}}=\frac{s C_{2} g_{m} A}{D(s)},
$$

where

$$
D(s)=s^{2} C_{1} C_{2}+s C_{2} g_{m} A+\frac{g_{m}}{R_{f}} .
$$

Angular pole frequency $\omega_{\mathrm{p}}$ of the filter can be adjusted by voltage-controlled $g_{\mathrm{m}}=1 / R_{\mathrm{f}}=1 / R$ and gain $A$ serves for electronic control of the pole quality factor $(Q)$. Both parameters $\left(\omega_{\mathrm{p}}, Q\right)$ are mutually independent as obvious from:

$$
\begin{gathered}
\omega_{p}=\frac{1}{R} \sqrt{\frac{1}{C_{1} C_{2}}}, \\
Q=\frac{1}{A} \sqrt{\frac{C_{1}}{C_{2}}} .
\end{gathered}
$$

Moreover, angular pole frequency of the band-pass filter can be tuned without disturbing the bandwidth (BW) of the BP filter by $R_{\mathrm{f}}$, because

$$
B W=\frac{g_{m} A}{C_{1}}
$$

\section{INFLUENCES OF GAIN INACCURACY OF ACTIVE DEVICES}

There are many non-idealities that have significant impact on the filter in more realistic scenario and their combined effect is usually not fully proven until real measurement of the constructed prototype is carried out. In this section we will focus on the most significant non-ideal aspect that forms the behaviour of the filter significantly (and the following section will present the measurement results covering all non-idealities compared with the simulation results).

According to (1), input current is mirrored or inverted as follows: $I_{+\mathrm{z}}=-I_{\mathrm{z}}=I_{\mathrm{zc}}=I_{\mathrm{f}}$. However, in the non-ideal case, there is non-unity gain between these terminals. The denominator (5) changes to

$$
\begin{gathered}
D^{\prime}(s)=s^{2} C_{1} C_{2}+s C_{2}\left(g_{m} A+\left(1+n_{12}\right) \frac{C_{2}}{R_{f}}\right)+ \\
+n_{11} \frac{g_{m}}{R_{f}}=0
\end{gathered}
$$

where $n_{11}$ is the transfer from $f$ terminal to $-z$ terminal and $n_{12}$ is the transfer from $f$ terminal to $z c$ terminal. In case of our behavioural model [24], [25], $n_{11}=-n_{12}=0.75$ [29] Therefore (9) changes to

$$
D^{\prime}(s)=s^{2} C_{1} C_{2}+s C_{2}\left(g_{m} A+\frac{0.25}{R_{f}}\right)+0.75 \frac{g_{m}}{R_{f}}=0 .
$$

Angular frequency, quality factor and bandwidth, i.e. (6)(8), should be recalculated with respect to (10). Since it represents just routine calculations, it is omitted in this paper. However, it is obvious that they will differ from (6)(8).

\section{Simulation AND MEASUREMENT REsults}

Behavioural model of ZC-VCCFDITA was tested in order to explore especially its particular tuning ranges in case of real on-board configuration. The first controllable parameter $R_{\mathrm{f}}$ is controlled by $V_{\text {set_Rf. Measured range of tuning starts }}$ from $485 \Omega\left(V_{\text {set_Rf }} \rightarrow 0 \mathrm{~V}\right)$ and ends on $2780 \Omega\left(V_{\text {set_Rf }}=\right.$ $-2 \mathrm{~V})$. The second tuneable parameter $A$ has measured minimum of $-39.9 \mathrm{~dB}\left(V_{\text {set } \mathrm{A}} \rightarrow 0 \mathrm{~V}\right)$ and maximum is $38.6 \mathrm{~dB}\left(V_{\text {set_A }}=-2 \mathrm{~V}\right)$. The last parameter $g_{\mathrm{m}}$ has been measured on both outputs, i.e. $+\mathrm{x}$ and $-\mathrm{x}$. Variation of these values is less than $1 \%$ in whole tuning range with minimum value of $0.2 \mathrm{uS}\left(V_{\text {set_gm }} \rightarrow 0 \mathrm{~V}\right)$ and maximum value of $3.07 \mathrm{mS}\left(V_{\text {set_gm }}=3.6 \mathrm{~V}\right)$.

Particular values of the filter were initially designed or calculated with respect to the above mentioned limits of behavioural model as follows. Theoretical tuning range of $f_{\mathrm{p}}=\omega_{\mathrm{p}} / 2 \pi=\{0.2 ; 1.06\} \mathrm{MHz}$ was obtained by voltagecontrolled simultaneous adjusting of $R_{\mathrm{f}}$ (by $V_{\text {set_Rf }}=\{-2 ;-$ $0.4\} \mathrm{V}$ ) and simultaneous control of $1 / g_{\mathrm{m}}$ (by $V_{\text {set gm }}=$ $\{0.388 ; 2.1\} \mathrm{V})$, with gained range $R=\{2780 ; 527\} \Omega$. Next, tuning range of $Q=\{0.5 ; 80\}$ was achieved by voltage-controlled adjusting of $A=\{0.061 ; 10\}$ tuned by $V_{\text {set_A }}=\{-1.5 ;-0.4\} \mathrm{V}$. Capacitor values were calculated as $C_{1}=1392 \mathrm{pF}$ and $C_{2}=59 \mathrm{pF}$, but with respect to real nodal parasitic capacitances (parallel combination of capacitances of all terminals connected to respective node), these capacitors were decreased to $C_{1}=1372 \mathrm{pF}$ (approximately $5 \mathrm{pF}$ per one connected terminal of ZC-VCCFDITA) and $C_{2}=52 \mathrm{pF}$. Real values (during measurements) were $C_{1}=1360 \mathrm{pF}$ and $C_{2}=39 \mathrm{pF}$, because of additional onboard parasitic capacitances having approximately $10 \mathrm{pF}$ in each node. 
The further three graphs compare the obtained measurement results with simulations under similar conditions, both prepared with above discussed behavioural model.

The first graph (Fig. 3) presents the $f_{\mathrm{p}}$ tuning over whole tuning range in the case of setting quite high $Q$ of BP filter. Table II includes a comparison of $f_{\mathrm{p}}$ and $Q$ with respect to the theoretical values, i.e. the values calculated with help of (6) and (7), non-ideal values calculated from (10), also the simulation results and finally measured characteristics. Measured pole frequency is slightly lower than expected in each case, quality factor is also lower than expected and increases with the pole frequency, together with pass-band gain that is slightly lower than expected, but all these values are in good agreement with the simulation results.

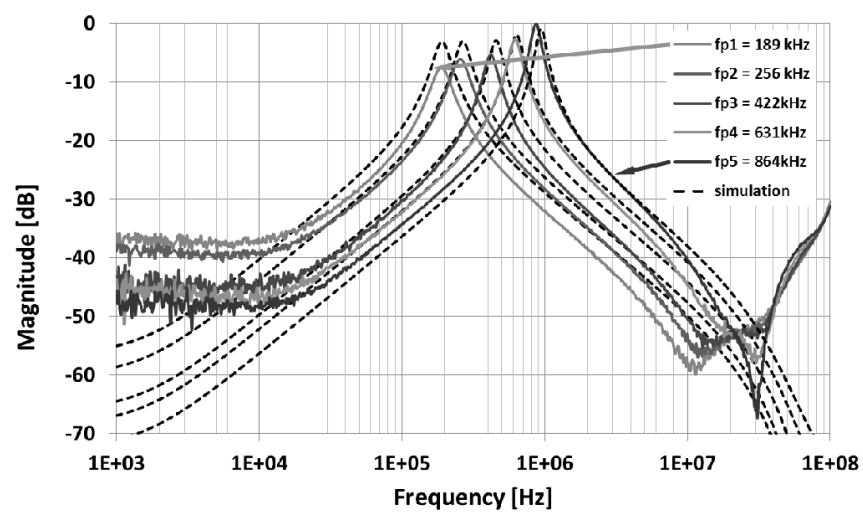

Fig. 3. Example results of electronically tuned $f_{\mathrm{p}}$ of the BP filter (measured values of $f_{\mathrm{p}}$ in legend) while keeping the $Q$ parameter constant.

TABLE II. COMPARISON OF OBTAINED RESULTS IN THE CASE OF INDEPENDENTLY TUNING THE POLE FREQUENCY (FIG. 3).

\begin{tabular}{|c|c|c|c|c|c|c|c|}
\hline 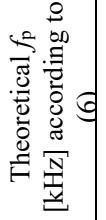 & 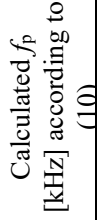 & 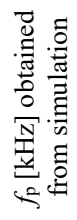 & 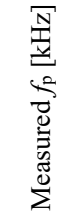 & 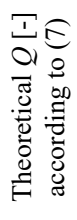 & 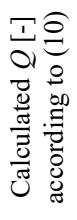 & 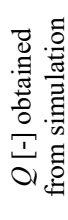 & 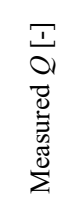 \\
\hline 200 & 191 & 192 & 189 & 8.4 & 5.3 & 3.9 & 3.2 \\
\hline 269 & 257 & 267 & 256 & 8.4 & 5.3 & 4.0 & 3.4 \\
\hline 462 & 442 & 450 & 422 & 8.4 & 5.3 & 4.8 & 4.7 \\
\hline 730 & 695 & 634 & 631 & 8.4 & 5.3 & 4.9 & 5.1 \\
\hline 1060 & 980 & 933 & 864 & 8.4 & 5.3 & 6.4 & 6.2 \\
\hline
\end{tabular}

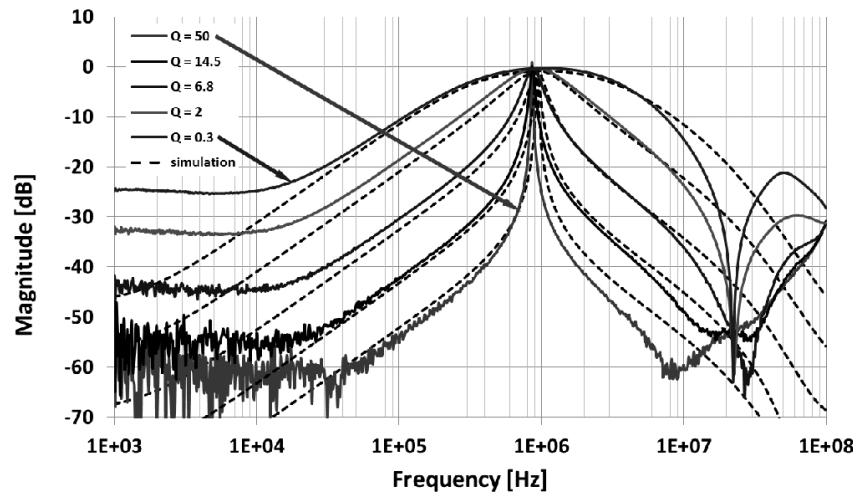

Fig. 4. Example results of electronically tuned $Q$ of the BP filter (measured values of $Q$ in legend) while keeping other parameters constant.

The second graph (Fig. 4) presents the $Q$ tuning over whole tuning range in case of setting the highest $f_{\mathrm{p}}$ of the BP filter $(1060 \mathrm{kHz}$ theoretically, but according to Table I,
$864 \mathrm{kHz}$ in measurement). Table III includes a comparison of $Q$ values for all scenarios in similar way as in Table II. Again, measured values of $Q$ are close to the expectations from simulations and slightly lower than expected from theory in case of very high values of $Q$. Nevertheless, the highest obtained value $(Q=50)$ is considered as very selective BP response.

TABLE III. COMPARISON OF OBTAINED RESULTS IN THE CASE OF INDEPENDENTLY TUNING THE QUALITY FACTOR (FIG. 4).

\begin{tabular}{|c|c|c|c|}
\hline $\begin{array}{c}\text { Theoretical } Q \\
{[-](7)}\end{array}$ & $\begin{array}{c}\text { Calculated } \\
Q[-](10)\end{array}$ & $\begin{array}{c}Q[-] \text { from } \\
\text { simulation }\end{array}$ & $\begin{array}{c}\text { Measured } Q \\
{[-]}\end{array}$ \\
\hline 0.5 & 0.5 & 0.6 & 0.4 \\
\hline 1.4 & 1.3 & 1.2 & 2 \\
\hline 5.3 & 4.0 & 4.2 & 6.8 \\
\hline 21.1 & 18.1 & 14.1 & 14.5 \\
\hline 80.0 & 62.3 & 45.3 & 50.0 \\
\hline
\end{tabular}

The last graph (Fig. 5) and Table IV presents the results of the $f_{\mathrm{p}}$ tuning while keeping the $B W$ parameter constant. It should be noted that required $B W$ together with $f_{\mathrm{p}}$ can be fine-tuned precisely, because of continuous (analogue) adjusting of both the control voltages ( $V_{\text {set_Rf }}$ and $\left.V_{\text {set_gm }}\right)$. Therefore, in final application it is required to tune not only one parameter as expected from the theoretical calculations, but also fine-tune other tuneable parameter(s) in order to reach the expected results.

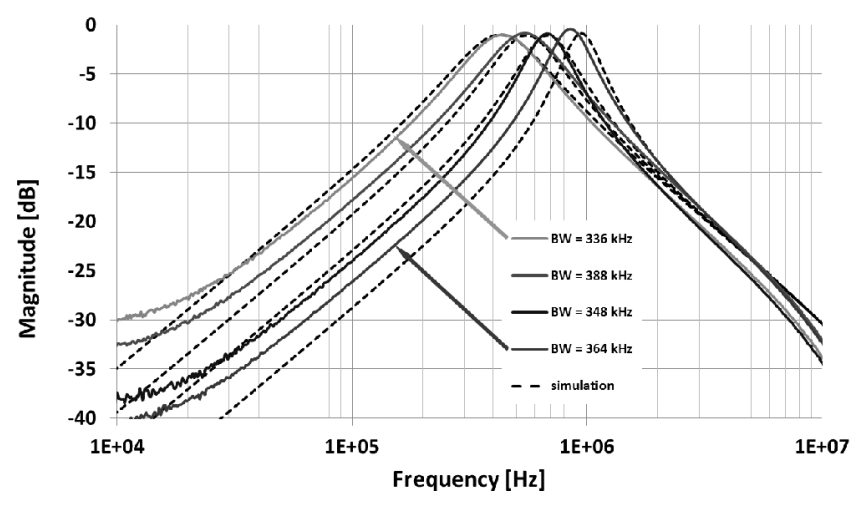

Fig. 5. Example results of electronically tuned $f_{\mathrm{p}}$ of the BP filter (measured values of $B W$ in legend) while keeping the $B W$ parameter constant.

TABLE IV. COMPARISON OF OBTAINED RESULTS IN THE CASE OF KEEPING THE BANDWIDTH CONSTANT (FIG. 5).

\begin{tabular}{|c|c|c|c|}
\hline $\begin{array}{c}\text { Theoretical } B W \\
{[\mathrm{kHz}](8)}\end{array}$ & $\begin{array}{c}\text { Calculated } \\
B W[\mathrm{kHz}](10)\end{array}$ & $\begin{array}{c}B W[\mathrm{kHz}] \\
\text { from } \\
\text { simulation }\end{array}$ & $\begin{array}{c}\text { Measured } B W \\
{[\mathrm{kHz}]}\end{array}$ \\
\hline 328 & 342 & 355 & 336 \\
\hline 328 & 355 & 363 & 388 \\
\hline 328 & 359 & 363 & 348 \\
\hline 328 & 386 & 361 & 364 \\
\hline
\end{tabular}

\section{CONCLUSIONS}

Behavioural model of ZC-VCCFDITA together with its application in controllable filter were presented in this paper. The main experimentally achieved features of the presented circuits are following: range of pole frequency tenability was tested from $189 \mathrm{kHz}$ to $864 \mathrm{kHz}$, quality factor adjusting was allowed from 0.4 to 50 by $V_{\text {set_A }}$ from $-1.5 \mathrm{~V}$ to $-0.4 \mathrm{~V}$ ( $A=0.06-10)$. Moreover, if $Q$ was 50 , actual $B W$ was only $17 \mathrm{kHz}$ at frequency of $864 \mathrm{kHz}$. The obtained results prove workability of the concept even in the stage, although there are some restrictions given by particular design of this active 
element, composed of commercially available devices that are not supposed to be used in high-frequency bands. It is obvious that the prepared model works satisfactorily almost up to $20 \mathrm{MHz}$ (parasitic poles and zeros). However, these results are promising and represent solid grounds for future on-chip implementation. In addition, some advanced approaches to CMOS subpart construction may improve the features of the used active device [32].

\section{REFERENCES}

[1] Y. Sun, J. K. Fidler, "Some design methods of OTA-C and CCII-RC filters", in Proc. IEE Colloquium on Digital and Analogue Filters and Filtering, London, 1993, pp. 1-8.

[2] Y. Sun, J. K. Fidler, "Structure generation of current-mode two integrator dual output-OTA grounded capacitor filters", IEEE Trans. Circuits Syst. II, Analog Digit. Signal Process., vol. 43, no. 9, pp. 659-663, 1996. [Online]. Available: http://dx.doi.org/10.1109/ 82.536762

[3] Y. Sun, J. K. Fidler, "Current-mode multiple-loop feedback filters using dual output OTAs and grounded capacitors", Int. J. Circuit Theory Appl., vol. 25, no. 2, pp. 69-80, 1997. [Online]. Available: http://dx.doi.org/10.1002/(SICI)1097-007X(199703/04)25:2<69:: AID-CTA950>3.0.CO;2-9

[4] T. Dostal, "Filters with multi-loop feedback structure in current mode", Radioengineering, vol. 12, no. 3, pp. 6-11, 2003.

[5] C. M. Chang, C. L. Hou, W. Y. Chung, J. W. Horng, C. K. Tu, "Analytical synthesis of high-order single-ended-input OTA grounded C all-pass and band-reject filter structures", IEEE Trans. Circuits Syst. I, Reg. Papers, vol. 53, no. 3, pp. 489-498, 2006. [Online]. Available: http://dx.doi.org/10.1109/TCSI.2005.859057

[6] S. Maheshwari, S. V. Singh, D. S. Chauhan, "Electronically tunable low-voltage mixed-mode universal biquad filter", IET Circuits Devices, Syst., vol. 5, no. 3, pp. 149-158, 2011. [Online]. Available: http://dx.doi.org/10.1049/iet-cds.2010.0061

[7] H. A. Alzaher, "A CMOS digitally programmable universal currentmode filter", IEEE Trans. Circuits Syst. II, Exp. Briefs, vol. 55, no. 8, pp. 758-762, 2008. [Online]. Available: http://dx.doi.org/10.1109/ TCSII.2008.921599

[8] A. Yesil, F. Kacar, "Electronically tunable resistorless mixed mode biquad filters", Radioengineering, vol. 22, no. 4, pp. 1016-1025, 2013.

[9] M. Kumngern, F. Khateb, P. Phasukkit, S. Tungjitkusolmun, S. Junnapiya, "ECCCII-based current-mode universal filter with orthogonal control of wo and Q", Radioengineering, vol. 23, no. 2, pp. 687-696, 2014.

[10] J. Koton, N. Herencsar, K. Vrba, B. Metin, "Voltage-mode multifunction filter with mutually independent $\mathrm{Q}$ and $\omega 0$ control feature using VDDDAs", Analog Integrated Circuits and Signal Processing, vol. 81, no. 1, pp. 53-60, 2014. [Online]. Available: http://dx.doi.org/10.1007/s10470-014-0323-z

[11] W. Tangsrirat, O. Channumsin, "High-input impedance voltage-mode multifunction filter using a single DDCCTA and grounded passive elements", Radioengineering, vol. 20, no. 4, pp. 905-910, 2011.

[12] F. Kacar, A. Yesil, H. Kuntman, "Current-mode biquad filters employing single FDCCII", Radioengineering, vol. 21, no. 4 pp. 1269-1278, 2012.

[13] N. Herencsar, O. Cicekoglu, R. Sotner, J. Koton, K. Vrba, "New resistorless tunable voltage-mode universal filter using single VDIBA", Analog Integrated Circuits and Signal Processing, vol. 76, no. 2, pp. 251-260, 2013. [Online]. Available: http://dx.doi.org/10.1007/s10470-013-0090-2

[14] O. Casas, R. Pallas-Areny, "Basics of analog differential filters", IEEE Trans. Instrum. Meas., vol. 45, no. 1, pp. 275-279, 1996. [Online]. Available: http://dx.doi.org/10.1109/19.481347

[15] M. A. Youssef, A. M. Soliman, "A novel CMOS realization of the differential input balanced output current operational amplifier and its applications", Analog Integrated Circuits and Signal Processing, vol. 44, no. 1, pp. 37-53, 2005. [Online]. Available: http://dx.doi.org/10.1007/s10470-005-1613-2

[16] M. O. Shaker, S. A. Mahmoud, A. M. Soliman, "New CMOS fullydifferential transconductor and application to a fully-differential Gm-
C filter", ETRI Journal, vol. 28, no. 2, 2006. [Online]. Available: http://dx.doi.org/10.4218/etrij.06.0105.0173

[17] M. Altun, H. Kuntman, "Design of a fully differential current mode operational amplifier with improved input-output impedances and its filter applications", International Journal of Electronics and Communications (AEU), vol. 62, no. 3, pp. 239-244, 2008. [Online]. Available: http://dx.doi.org/10.1016/j.aeue.2007.03.020

[18] E. A. Sobhy, A. M. Soliman, "Realizations of fully differential voltage second generation current conveyor with an Application", Int. J. Circuit Theory Appl., vol. 38, no. 5, pp. 441-452, 2010. [Online]. Available: http://dx.doi.org/10.1002/cta.566

[19] D. Biolek, R. Senani, V. Biolkova, Z. Kolka, "Active elements for analog signal processing: classification, review and new proposals", Radioengineering, vol. 17, no. 4, pp. 15-32, 2008.

[20] R. Sotner, J. Petrzela, J. Slezak, "Current-controlled current-mode universal biquad employing multi-output transconductors", Radioengineering, vol. 18, no. 3, pp. 285-294, 2009.

[21] R. Sotner, B. Sevcik, L. Brancik, T. Dostal, "Multifunctional adjustable biquadratic active RC filters: design approach by modification of corresponding signal flow graphs", Przeglad Elektrotechniczny, vol. 87, no. 2, pp. 225-229, 2011.

[22] J. Jerabek, J. Koton, R. Sotner, K. Vrba, "Comparison of fullydifferential and single-ended current-mode band- pass filters with current active elements", in Proc. of 7th Int. Conf. Electrical and Electronics Engineering, (ELECO 2011), Bursa, 2011, pp. 100-104.

[23] J. Jerabek, J. Koton, R. Sotner, K. Vrba, “Adjustable band-pass filter with current active elements: two fully-differential and single-ended solutions", Analog Integrated Circuits and Signal Processing, vol. 74, no. 1, pp. 129-139, 2013. [Online]. Available: http://dx.doi.org/10.1007/s10470-012-9942-4

[24] J. Jerabek, R. Sotner, N. Herencsar, W. Jaikla, K. Vrba, "Behavioral model for Z- copy voltage controlled current follower differential input transconductance amplifier and its features", in Proc. 38th Int Conf. Telecommunication and Signal Processing (TSP 2015), Prague, 2015, pp. 703-707. [Online]. Available: http://dx.doi.org/ 10.1109/TSP.2015.7296354

[25] R. Sotner, J. Jerabek, N. Herencsar, J-W. Horng, K. Vrba, "Electronically linearly voltage controlled second-order harmonic oscillator with multiples of pi/ 4 phase shifts", in Proc. 38th Int Conf. Telecommunication and Signal Processing (TSP 2015), Praha 2015, pp. 708-712. [Online]. Available: http://dx.doi.org/10.1109/ TSP.2015.7296355

[26] N. Herencsar, J. Koton, K. Vrba, A. Lahiri, "Single GCFDITA and grounded passive elements based general topology for analog signal processing applications", in Proc. 11th Int. Conf. Networks, (ICN 2012), Saint Gilles, 2012, pp. 59-62.

[27] N. Herencsar, J. Koton, I. Lattenberg, K. Vrba, "Signal-flow graphs for current-mode universal filter design using current follower transconductance amplifiers (CFTAs)", in Proc. Int. Conf. On Applied Electronics (APPEL 2008), Pilsen, 2008, pp. 69-72.

[28] A. Fabre, O. Saaid, F. Wiest, C. Boucheron, "High frequency applications based on a new current controlled conveyor", IEEE Trans. Circuits Syst. I, Fundam. Theory Appl., vol. 43, no. 2, pp. 8291, 1996. [Online]. Available: http://dx.doi.org/10.1109/81.486430

[29] R. Sotner, A. Kartci, J, Jerabek, N. Herencsar, T. Dostal, K. Vrba, "An additional approach to model current followers and amplifiers with electronically controllable parameters from commercially available ICs", Measurement Science Review, vol. 12, no. 6, pp, 255265, 2012. [Online]. Available: http://dx.doi.org/10.2478/v10048012-0035-4

[30] R. L. Geiger, E. Sanchez-Sinencio, "Active filter design using operational transconductance amplifiers: a tutorial", IEEE Circuits Devices Mag., vol. 1, no. 2, pp. 20-32, 1985. [Online]. Available: http://dx.doi.org/10.1109/MCD.1985.6311946

[31] R. Sotner, Z. Hrubos, N. Herencsar, J. Jerabek, T. Dostal, K. Vrba "Precise electronically adjustable oscillator suitable for quadrature signal generation employing active elements with current and voltage gain control", Circuits, Systems and Signal Processing, vol. 33, no. 1, pp. 1-35, 2014. [Online]. Available: http://dx.doi.org/10.1007/ s00034-013-9623-2

[32] O. G. Sokmen, H. Ercan, S. A. Tekin, M. Alci, “A Novel low voltage low power OTA based on level shifter current mirror", Elektronika ir Elektrotechnika, vol. 21, no. 2, pp. 39-43, 2015. [Online]. Available: http://dx.doi.org/10.5755/j01.eee.21.2.11511 